\title{
A Fractal Approach to Goose Down Structure
}

\author{
Jing GAO ${ }^{a}$, Ning PAN ${ }^{b}$, Weidong YU ${ }^{a}$ \\ ${ }^{a}$ Textile College, Donghua University, West Yan'an Road 1882, Shanghai 200051, China \\ ${ }^{b}$ Biological and Agricultural Engineerings Department, University of California, Davis. California 95616. \\ U.S.A.
}

\begin{abstract}
Goose down has superior thermal insulating property as a filler materials used widely in various insulated textile products. In this paper the fractal structure of the goose down is theoretically analyzed, its fractal dimension is calculated as 1.66, which is very near to the Golden Mean, 1.618, revealing certain optimal structure of goose down.
\end{abstract}

Keywords: fractal, fractal dimension, goose down, textile, Golden mean

I want to know how God created this world. I am not interested in this or that phenomenon, in the spectrum of this or that element. I want to know His thoughts. -Albert Einstein

What miracles exist in mathematics! According to my theory, the golden Proportion of the ancient Greeks gives the minimal power condition of the butadiene molecule. -Richard Feynman

\section{Introduction}

Golden proportion is also exited in goose down, which is a layer of undercoat growing on the cuticular of fowl, such as goose, duck and swan etc. Goose down and duck down are the most usual down, which show themselves soft and fine globosity. For long time, down has been using as the best filler material of beddings and outerwear withstanding cold climates because of its superior thermal insulating property. The investigation on the configuration characters and thermal insulation properties of down have never stopped, and there are several publications [1 3] on the morphology and structure, as well as physico-chemical properties, of the down. However, no attempt is made so far by fractal approach to study heat transfer in single goose down.

Fractal approach might become a new ramification in textile engineering. Fractal structures are widely appeared in various textile productions, natural or artificial. The specific characteristics of fractal structures, such as the self-similarity and the fractal dimension " $D_{f}$ ", endow them with special physical properties. In this paper we will first apply fractal geometry to goose down, and our analysis shows that the goose down is of optimal fractal construction.

\section{Fractal Structure of Goose Down}

Figure1 (a) shows a usual goose down as soft and fine globosity by common numeral camera. However, magnified in the scanning electron microscope, goose down will show itself a large tree shape. The whole down consists of a short central nucleus from which a number of branches diverge at a wide range of orientations (Figure1b). Comparing with the down branch, the down nucleus is very small which can be considered as a dot in the macroscopical picture. On some main branches, analogous secondary branches will shoot out, and keep the same structures with the main branches (Figure1c). Also each branch carries in turn a large number of fibrils all of which diverge from the branches at about $45^{\circ}$ (Figure1d). The down branches and down fibrils constitute the main body of down. Further many little triangle nodes and 
crotches diverge from the fibrils to hold in place a single crossing fibril (Figure1e).

(a) Individual down cluster
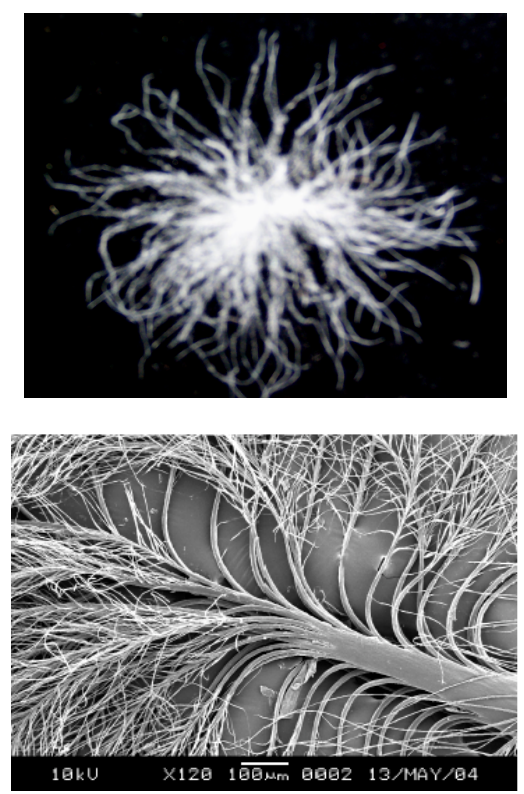

(b) Down branches diverging from down nucleus.

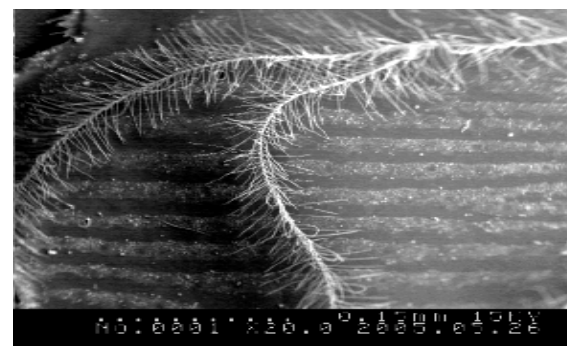

(c) Secondary branch diverging from main branch.

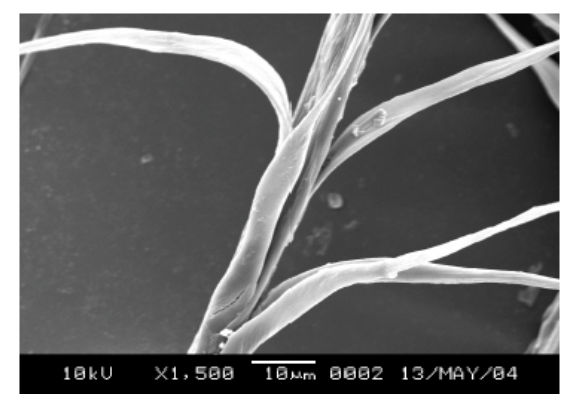

(d) Down fibrils diverging from down branch.

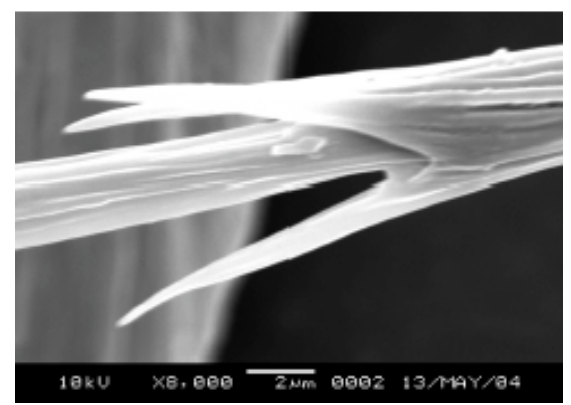

(e) Crotch nodes on down fibril.

Figure 1. Fractal "tree" structure of down.
Of the many grades of structural divarications in goose down, each grade of divarications possesses a statistical self-similarity with other grades or with the whole cluster. Whether magnifying or minifying the figures, the configuration characters remain invariant. In other words, the goose down has evident "fractal tree" structure.

Simulating the morphologic structure and structure parameters of goose down, the computer generating graphics after fractal three times is showed in Figure 2.

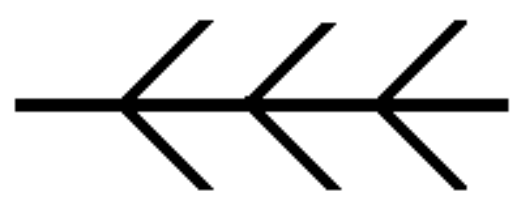

(a)

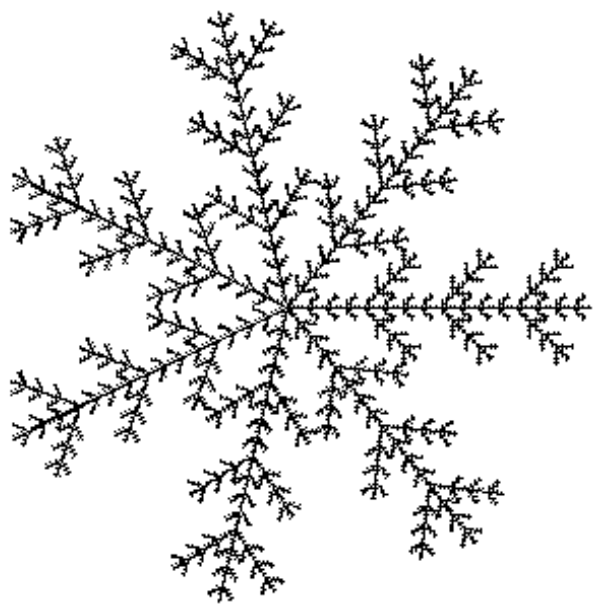

(b)

Figure 2. Fractal tree of down at 1-iteration-level (a), and three levels of iteration (b).

Fractal approach is widely used in engineering analysis $[4,5,6]$. The fractal dimension $D_{f}$ of triple fractal unit as seen in Figure 3 and 4 of the

goose down is $D_{f}=\ln 10 / \ln 4 \approx 1.66$

The fractal dimension is 1.66 , very near to the Golden Mean, 1.618, revealing the optimal structure in goose down. Iovane [7] showed the importance of the golden mean in respect to the large scale structures in our Universe; Weiss et al.[8] illustrated that the principle of information coding by the brain seems to be based on the golden mean. Keshavarzi et al. [9] found that the 
fractal dimension of turbulence is relative to 1.618; He and Zhang[10] applied the golden mean to biomechanics.

The main application of golden mean in the E-infinity theory [11-18] shows miraculous exactness compared to experimental measurement, especially in determining the theoretically coupling constants and the mass spectrum of the standard model of elementary particles. A new mathematical direction called Harmony mathematics based on Golden mean is systematically established [19-23].

\section{Principle of heat transfer modeling}

Zmeskal et al.[6] studied thermal properties of bodies in fractal and cantorian physics; Haba et al.[5] studied the influence of the electrical parameters on the input impedance of a fractal structure realized in silicon. Hereby we follow the basic approach proposed in [5].

Suppose that every elementary cell in fractal-generating-unit (Figure 2a) has equal thermal resistance, $\mathrm{R}$, then the equivalent thermal layout of fractal unit is showed in Figure 3.

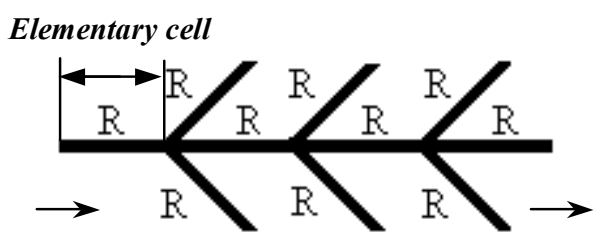

Figure 3. Elementary cell in fractal unit.

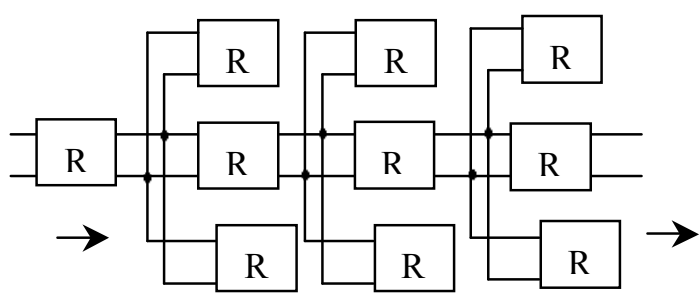

Figure 4. Equivalent thermal layout of fractal unit.

Thermal resistance has analogous transferring-properties with electric resistance, and keeps to the same series-parallel connection rules. In other words, the thermal resistance of the down during heat transfer is equivalent to the illustration in Figure 4.

So the overall thermal resistance for a triple fractal unit is:

$$
R_{u}=R+\frac{1}{\frac{1}{R}+\frac{1}{R}+\frac{1}{R+\frac{1}{\frac{1}{R}+\frac{1}{R}+\frac{1}{R+\frac{1}{\frac{1}{R}+\frac{1}{R}+\frac{1}{R}}}}}}=\frac{56}{41} R
$$

The thermal resistance of the fractal-generating-unit is the basic physical characters of goose down, which will affect the whole cluster thermal transferring and fiber assembly thermal insulation. This will be demonstrated in a later report.

\section{Conclusions}

This study on the morphological structure of the goose down has showed that the down fibers have typical fractal structure. The many different grades divarications in down have obvious statistical self-similarity. Magnifying or minifying the down, the configuration characters remain invariant. The fractal dimension " $D_{f}$ " of goose down is 1.66, close to the Gold Mean, 1.618 , revealing the optimal structure in goose down. The optimal fractal dimension also dictates the heat transferring properties of down fibers. In fractal-generating-unit, thermal resistance superimposes according to the series-parallel connection rules, and be 1.366 times of the thermal resistance of elementary cell, which presumably ensure the excellent thermal insulations of the goose down and will be discussed in detail in our following paper.

Moreover according to the predicted thermal transferring properties of the fractal-generating-unit, the thermal resistance of a whole down and down assemblies. All of which requires further studies and will be reported subsequently.

\section{Acknowledgements}

The author expresses her sincere gratitude to the Professor Jihuan HE of College of Science in Donghua University for his unwearied guide and assistance in mathematics and fractal theory. 


\section{References}

[1] WU A., SONG Xiu. Structure and Properties of Feather And Down, Journal of China Textile University, 2(1990) 94-98.

[2] JIN Y, LI W. Study on the Structure and Characteristics of Natural Protein Fibers, Wool Textile Journal, 1(2000)23-26.

[3] Skelton J, Dent R, Donovan JG, The Thermal and Mechanical Properties of Down. Proceedings of The 7th International Wool Textile Research Conference, 3(1985)264-273.

[4] Gabrys E, Rybaczuk M. Blood flow simulation through fractal models of circulatory system, Chaos, Solitons and Fractals,27(1)(2006)1-7.

[5] Haba TC, Ablart G, Camps T, Olive F. Influence impendance of a fractal structure realized on silicon, Chaos, Solitons and Fractals, 24(2005)479-490.

[6] Zmeskal O, Buchnicek M, Vala M. Thermal properties of bodies in fractal and cantorian physics, Chaos, Solitons and Fractals, 25(5) (2005)941-954.

[7] Iovane, G. Varying G, accelerating Universe, and other relevant consequences of a stochastic self-similar and fractal Universe, Chaos, Solitons and Fractals, 20(4) (2004)657-667,.

[8] Weiss H, The golden mean as clock cycle of brain waves, Chaos, Solitons and Fractals, 18(4)(2003)643-652.

[9] Keshavarzi AR, Ziaei AN, Homayoun E, Shirvani A. Fractal Markovian scaling of turbulent bursting process in open channel flow, 25(2)(2005)307-318.

[10] He JH. Fifth dimension of life and the $4 / 5$ allometric scaling law for human brain, Cell Biology International, 28(11)(2004) 809-815.

[11] El Naschie MS. On a fuzzy Kahler-like manifold which is consistent with the two slit experiment, International Journal of Nonlinear Sciences and Numerical Simulation. 6(2)(2005) 95-98.
[12] El Naschie MS. Transfinite electrical networks, spinoral varieties and gravity Q bits, International Journal of Nonlinear Sciences and Numerical Simulation. 5(3) (2004)191-197.

[13] El Naschie MS. Einstein in a complex time some very personal thoughts about E-infinity theory and modern physics, International Journal of Nonlinear Sciences and Numerical Simulation. 6(3)(2005)331-333.

[14] Goldfain E. Complex Dynamics and the High-energy Regime of Quantum Field Theory, International Journal of Nonlinear Sciences and Numerical Simulation. 6(3)(2005)223-234.

[15] He JH. Application of E-infinity theory to biology, Chaos, Solitons and Fractals, 28(2)(2006), 285-289.

[16] El Naschie MS. A review of E infinity theory and the mass spectrum of high energy particle physics, Chaos, Solitons and Fractals, 19(1)(2004)209-236.

[17] El Naschie MS. A guide to the mathematics of E-infinity Cantorian spacetime theory. Chaos, Solitons and Fractals, 25(2005)955-964.

[18] El Naschie MS. Elementary number theory in superstrings, loop quantum mechanics, twistors and E-infinity high energy physics, Chaos, Solitons and Fractals, 27(2)(2006)297-330.

[19] Stakhov A. Fundamental of a new kind of mathematics based on the Golden Section, Chaos, Solitons and Fractals, 27(5)(2006)1124-1146.

[20] Stakhov A, Rozin B. Theory of Binet formulas for Fibonacci and Lucas p-numbers, Chaos, Solitons and Fractals, 27(5)(2006)1162-1177.

[21]Stakhov A, Rozin B. The Golden shofar,26(3)(2005)677-684.

[22] Stakhov A, Rozin B. On a new class of hyperbolic functions, Chaos, Solitons and Fractals, 27(5)(2006) 1415-1421. 